\title{
Acute kidney injury in COVID-19: a case-report
}

\author{
Marco Di Mauro ${ }^{1}$ Marco Giuseppe Migliaccio ${ }^{1} \cdot$ Riccardo Ricciolino $^{1} \cdot$ Giorgio Spiniello $^{1} \cdot$ Vincenzo Carfora $^{1}$. \\ Nicoletta Verde ${ }^{1} \cdot$ Filiberto Fausto Mottola $^{1} \cdot$ Nicola Coppola $^{2}$
}

Received: 27 July 2020 / Accepted: 31 August 2020 / Published online: 8 September 2020

(c) Springer Nature B.V. 2020

Editor,

A 81-year-old-Caucasian- female with a history of rheumatoid arthritis in chronic treatment methotrexate and metilprednisolone showed fever and chills partially responsive to antibiotic therapy, and dry cough. Since the persistence of the symptoms, she underwent an oropharyngeal swab for SARS-CoV-2-RNA, resulted positive and was hospitalized at our COVID-19 unit. A thoracic CT scan showed bilateral interstitial pneumonia with ground glass opacities. At the admission, the patient didn't need oxygen therapy, and $\mathrm{PaO} 2 / \mathrm{FiO} 2$ at haemogasanalysis was 319 . Her clinical parameters and lab tests were in the normal range (Table 1), except for mild anemia and high D-dimer. A therapy with lopinavir/ritonavir, hydroxychloroquine, enoxaparine was started. During the observation, at day 4 from hospitalization, she experienced progressive dyspnoea and hypoxemia despite a respiratory support with Venturi Mask $\left(\mathrm{FiO}_{2}\right.$ 24/28\%). Suspecting pulmonary embolism (PE), a thoracic angio-CT scan was performed, resulted negative for PE, but suggestive of an interstitial involvement more severe than the previous CT. Adjunctive therapy with tocilizumab and metilprednisolone was started. During the following days, the patient developed hypotension, oliguria and impaired renal function, with the lower glomerular filtration rate (GFR) of $16 \mathrm{~mL} / \mathrm{min}$, corresponding to a serum creatinine

Marco Di Mauro and Marco Giuseppe Migliaccio contributed equally to the paper.

Nicola Coppola

nicola.coppola@unicampania.it

1 Department of Translational Medical Sciences, University of Campania “Luigi Vanvitelli”, Naples, Italy

2 Section of Infectious Diseases, Department of Mental Health and Public Medicine, Infectious Diseases Unit, University of Campania "Luigi Vanvitelli", Via L. Armanni 5, 80131 Naples, Italy
(SCr) of $2.7 \mathrm{mg} / \mathrm{dL}, 3$ days after the angio-CT scan (day 7 from hospitalization, Table 1).

So, a contrast induced nephropathy (CIN) was suspected, and intravenous hydration therapy started. The patient did not drink any water, and also presented clinical signs of dehydration. Urinalysis was normal. She showed a restoration of a normal diuresis and improvement of renal function ( $\mathrm{SCr}$ and GFR), although never as normal as before the admission. She also developed unexplained anemia and thrombocytopenia, initially interpreted as related to enoxaparine, but more probably related to COVID-19.

After 30 days of hospitalization, and two swab test resulted negative for SARS-CoV2, the patient was discharged in a good respiratory and clinical condition.

Analyzing the possible causes of acute kidney injury (AKI), we can reasonably consider it as a CIN, for the onset of the renal function impairment occurred 3 days after intravenous contrast administration [1]. Rheumatoid arthritis (RA) or other causes of renal damage, as glomerulonephritis, seem to be unlikely, respectively due to the good control of RA and to the absence of worsening hypertension, proteinuria or hematuria caused by an acute glomerulonephritis.

The progression of renal damage cannot be attributed to the radiocontrast agent only: in fact, despite the restoration of a normal diuresis and the improvement of kidney function, $\mathrm{SCr}$ and GFR never returned to pre-hospitalization values, as we could usually expect in a CIN treated properly, and without a previous history of chronic kidney disease [2].

Furthermore, marked hypotension and the simultaneous deterioration of respiratory function suggested COVID-19 infection may have played a role [3, 4]. Increasing of LDH, D-Dimer and thrombocytopenia suggests a high inflammatory response to the infection. We can suppose that inflammation and immune response played an important role in the development of AKI, along with CIN [5-7].

The present clinical case suggests that during COVID-19 AKI may occur because of different causes that the physician should identify for a good management. 
Table 1 Biochemical data at admission and at day 7 of hospitalization

\begin{tabular}{lll}
\hline & At admission & Day 7 after admission \\
\hline Haemoglobin & $11.8 \mathrm{~g} / \mathrm{dL}$ & $9.1 \mathrm{~g} / \mathrm{dL}$ \\
White blood cells & $4.46 \times 10^{3} / \mathrm{mm}^{3}$ & $3.44 \times 10^{3} / \mathrm{mm}^{3}$ \\
Platelets & $132 \times 10^{3} / \mathrm{mm}^{3}$ & $248 \times 10^{3} / \mathrm{mm}^{3}$ \\
Serum creatinine & $0.8 \mathrm{mg} / \mathrm{dL}$ & $2.7 \mathrm{mg} / \mathrm{dL}$ \\
Urea & $49 \mathrm{mg} / \mathrm{dL}$ & $103 \mathrm{mg} / \mathrm{dL}$ \\
$\mathrm{Na}^{+}$ & $137 \mathrm{mEq} / \mathrm{L}$ & $133 \mathrm{mEq} / \mathrm{L}$ \\
$\mathrm{K}^{+}$ & $4.3 \mathrm{mEq} / \mathrm{L}$ & $3.8 \mathrm{mEq} / \mathrm{L}$ \\
Lactate dehydrogenase & $376 \mathrm{U} / \mathrm{L}$ & $354 \mathrm{U} / \mathrm{L}$ \\
D-dimer & $33 \mu \mathrm{g} / \mathrm{mL}-55 \times$ & $23 \mathrm{mcg} / \mathrm{mL}-38 \times$ \\
\hline
\end{tabular}

\section{References}

1. Mohammed NM, Mahfouz A, Achkar K, Rafie IM, Hajar R (2013) Contrast-induced nephropathy. Heart Views 14(3):106-116. https ://doi.org/10.4103/1995-705X.125926
2. Ali A, Bhan C, Malik MB, Ahmad MQ, Sami SA (2018) The prevention and management of contrast-induced acute kidney injury: a mini-review of the literature. Cureus 10(9):e3284. https://doi. org/10.7759/cureus.3284 (Published 2018 Sep 11)

3. Diao B et al (2020) Human kidney is a target for novel severe acute respiratory syndrome coronavirus 2 (SARS-CoV-2) infection. medRxiv. https://doi.org/10.1101/2020.03.04.20031120

4. Pei G et al. (2020) Renal involvement and early prognosis in patients with COVID-19 Pneumonia. J Am Soc Nephrol 2019:ASN.2020030276

5. Chousterman BG, Swirski FK, Weber GF (2017) Cytokine storm and sepsis disease pathogenesis. Semin Immunopathol. https:// doi.org/10.1007/s00281-017-0639-8

6. Husain-Syed F, Slutsky AS, Ronco C (2016) Lung-kidney crosstalk in the critically ill patient. Am J Respir Crit Care Med. https ://doi.org/10.1164/rccm.201602-0420CP

7. Gralinski LE et al (2018) Complement activation contributes to severe acute respiratory syndrome coronavirus pathogenesis. MBio. https://doi.org/10.1128/mBio.01753-18

Publisher's Note Springer Nature remains neutral with regard to jurisdictional claims in published maps and institutional affiliations. 